\title{
Correction to: Elastic, Viscoelastic and Fibril-Reinforced Poroelastic Material Properties of Healthy and Osteoarthritic Human Tibial Cartilage
}

\author{
Mohammadhossein Ebrahimi (D, ${ }^{1,2}$ Simo Ojanen, ${ }^{1,2}$ Ali Mohammadi, ${ }^{1}$ \\ Mikko A. Finnilä, ${ }^{2}$ Antti Joukainen, ${ }^{3}$ Heikki Kröger, ${ }^{3}$ \\ Simo SaArakkala, ${ }^{2}$ Rami K. Korhonen, ${ }^{1}$ \\ and Petri TANSKA ${ }^{1}$
}

${ }^{1}$ Department of Applied Physics, University of Eastern Finland, POB 1627, 70211 Kuopio, Finland; ${ }^{2}$ Research Unit of Medical Imaging, Physics and Technology, Faculty of Medicine, University of Oulu, Oulu, Finland; and ${ }^{3}$ Kuopio University Hospital, Kuopio, Finland

(Published online 6 October 2021)

Correction to: Annals of Biomedical Engineering, Vol. 47, No. 4, April 2019 (ㄷ 2019) pp. 953-966 https://doi.org/10.1007/s10439-019-02213-4

This correction is to update Equation 2. In Eq. (2), the Neo-Hookean hyperelastic material formulation reported for the non-fibrillar matrix was not the actual formulation used in our material model. The correct formulation is:

$$
\boldsymbol{\sigma}_{\mathrm{nf}}=\frac{1}{2} K_{\mathrm{nf}}\left(J-J^{-1}\right) \mathbf{I}+\frac{G_{\mathrm{nf}}}{J}\left(\mathbf{F} \mathbf{F}^{\mathrm{T}}-J^{\frac{2}{3}} \mathbf{I}\right),
$$

where $\sigma_{\mathrm{nf}}$ is the stress tensor of the non-fibrillar matrix, $G_{\mathrm{nf}}$ and $K_{\mathrm{nf}}$ are the shear and bulk moduli of the non- fibrillar matrix, respectively, $\boldsymbol{F}$ is the deformation gradient tensor, $J$ is the determinant of the $\boldsymbol{F}$ and $\boldsymbol{I}$ is the unit tensor.

This mistake was authors' typing error. The corrected equation was used in all original analyses, thus, this correction does not affect the results, nor does it affect the conclusions made in the article.

The original article has been corrected.

Publisher's Note Springer Nature remains neutral with regard to jurisdictional claims in published maps and institutional affiliations.

Address correspondence to Mohammadhossein Ebrahimi, Department of Applied Physics, University of Eastern Finland, POB 1627, 70211 Kuopio, Finland. Electronic mail: mohammadhossein. ebrahimi@uef.fi; ebrahimi.bioengineering@gmail.com

The original article can be found online at https://doi.org/10. 1007/s10439-019-02213-4. 